\title{
Scalar Sector Phenomenology of Three-Loop Radiative Neutrino Mass Models
}

\author{
Amine Ahriche, ${ }^{a, b}{ }_{1}$ Kristian L. McDonald ${ }^{c} 2_{2}$ and Salah Nasri ${ }^{d .3}$ \\ a Department of Physics, University of Jijel, PB 98 Ouled Aissa, DZ-18000 Jijel, Algeria \\ $b$ The Abdus Salam International Centre for Theoretical Physics, Strada Costiera 11, \\ I-34014, Trieste, Italy \\ ${ }^{c}$ ARC Centre of Excellence for Particle Physics at the Terascale, \\ School of Physics, The University of Sydney, NSW 2006, Australia \\ ${ }^{d}$ Physics Department, UAE University, POB 17551, Al Ain, United Arab Emirates
}

\begin{abstract}
We perform a phenomenological study of the scalar sector of two models that generate neutrino mass at the three-loop level and contain viable dark matter candidates. Both models contain a charged singlet scalar and a larger scalar multiplet (triplet or quintuplet). We investigate the effect of the extra scalars on the Higgs mass and analyze the modifications to the triple Higgs coupling. The new scalars can give observable changes to the Higgs decay channel $h \rightarrow \gamma \gamma$ and, furthermore, we find that the electroweak phase transition becomes strongly first-order in large regions of parameter space.
\end{abstract}

PACS: 12.60.Fr, 12.60.-I, 95.35.+d.

\footnotetext{
${ }^{1}$ aahriche@ictp.it

${ }^{2}$ klmcd@physics.usyd.edu.au

${ }^{3}$ snasri@uaeu.ac.ae
} 


\section{Introduction}

The Standard Model (SM) of particle physics is a spectacularly successful theory that stands as one of the truly great scientific achievements. Despite this success, however, the theory possesses a number of short-comings, suggesting it will likely require extensions and/or modifications in the future. The most obvious motivation for extending the SM is the need to incorporate gravity. However, a lack of present-day experimental guidance makes the pursuit of the theory of quantum gravity an incredibly challenging task.

Additional evidence that the SM is incomplete comes from the experimental observation of neutrino mixing and the need to explain the missing gravitating (i.e. dark) matter, required on galactic scales. These puzzles motivate the addition of new particle species to the SM and there is much hope that such new species will manifest in future experiments (in particular at Run II of the LHC). The neutrino mass and dark matter (DM) problems have stimulated much research and there are multiple candidate solutions one can pursue. The problems may have independent solutions, though it seems reasonable to ask whether the two puzzles could share a unified or common solution. Could the neutrino mass and DM problems be related?

In 2002, Krauss, Nasri and Trodden (KNT) proposed a simple extension of the SM model that admits a relationship between the existence of DM and the origin of neutrino mass [1]. In this approach, one adds new fields to the SM, such that neutrino mass is generated radiatively at the three-loop level, with one of the particles propagating in the mass diagram being a DM candidate. The model employs two charged singlet scalars, $S_{1,2}^{+}$, and three generations of gauge-singlet fermions $N$. A $Z_{2}$ symmetry with action $\left\{S_{2}^{+}, N\right\} \rightarrow\left\{-S_{2}^{+},-N\right\}$ is also imposed. This ensures stability of the lightest fermion $N$, thereby giving a DM candidate, and also prevents a coupling between SM neutrinos and $N$, which would otherwise generate tree-level neutrino masses. The result is a type of unified description for the origin of neutrino mass and DM, with the removal of the DM candidate simultaneously turning off neutrino mass 4

In recent years, a number of basic generalizations of the KNT model have appeared. In one such model (hereafter 'the triplet model'), the singlet fields $S_{2}^{+}$and $N$ are replaced by $S U(2)_{L}$ triplets [6]. This model retains the $Z_{2}$ symmetry to ensure DM stability and prevent tree-level neutrino masses, and gives a viable alternative unified framework for the DM and neutrino mass problems. A further generalization exchanges the singlet fields $S_{2}^{+}$and $N$ for $S U(2)_{L}$ quintuplet fields [7]. This model (hereafter 'the quintuplet

\footnotetext{
${ }^{4}$ For recent studies of the KNT model see Refs. [2, 3, 4, 5].
} 
model') does not require the $Z_{2}$ symmetry in order to prevent tree-level neutrino masses, and is a viable theory for radiative neutrino mass, independent of DM considerations. Interestingly, the most-general Lagrangian for the model contains a single $Z_{2}$-breaking coupling $\lambda$. When taken small, $\lambda \ll 1$, the model gives a long-lived DM candidate, while turning $\lambda$ off completely, $\lambda \rightarrow 0$, activates a $Z_{2}$ symmetry and gives absolutely stable DM. Thus, the quintuplet model is a viable model of radiative neutrino mass, with or without DM.

Due to the presence of larger multiplets with non-trivial $S U(2)$ charges in both the triplet and quintuplet models, the phenomenology of the models is rather rich. In the present work, we extend the analysis of Refs. [6, [7] and undertake a more extensive study of the phenomenology of both models. We investigate the effect of the triplet and quintuplet scalars on both the Higgs mass and the triple Higgs coupling, showing that the latter can experience sizable modifications. We also study the effect of the new multiplets on the Higgs decay channels $h \rightarrow \gamma \gamma, \gamma Z$. Our work shows that, e.g. observable changes are expected to $\mathcal{B}(h \rightarrow \gamma \gamma)$, with some regions of parameter space already excluded for the triplet model. The effect of the enlarged scalar sector on the electroweak phase transition is also analyzed, revealing a tendency for a strongly first-order phase transition in large regions of parameter space.

Before proceeding we note that further generalizations of the KNT model are possible. Ref. [8] presented colored generalizations and other related three-loop models that employ slightly modified loop topologies. A septuplet generalization of the KNT model was proposed in Ref. 9]. This had the interesting feature of automatically containing an absolutely stable DM candidate, without requiring a new symmetry. A minimal scaleinvariant implementation was also recently studied [10]. More generally, a number of authors have studied connections between radiative neutrino mass and DM in recent years, see e.g. Refs. [11]-[15].

This paper is structured as follows. In Section 2, we outline the triplet and quintuplet models, describing some key features and elucidating some stability constraints on the scalar potentials. We study the influence of the new multiplets on the Higgs mass and the triple Higgs coupling in Section 3. The electroweak phase transition is considered in Section 4 and we turn to the Higgs decay channels $h \rightarrow \gamma \gamma$ and $h \rightarrow \gamma Z$ in Section 5 , Conclusions are presented in Section 6. 


\section{Three-Loop Radiative Neutrino Masses}

The SM employs the gauge symmetry $\mathcal{G}_{S M}=S U(3)_{c} \times S U(2)_{L} \times U(1)_{Y}$. In this work, we consider extensions of the SM that include the charged singlet scalar $S \sim(1,1,2)$, the scalar multiplet $T \sim(1,2 n+1,2)$ and three generations of chiral beyond-SM fermions, $\mathcal{F}_{i} \sim(1,2 n+1,0)$, where $i=1,2,3$, labels generations and numbers in parenthesis denote charges under $\mathcal{G}_{S M}$. We use the integer $n=0,1,2$, to label the distinct models. The case with $n=0$ is the KNT model, for which all beyond-SM fields are $S U(2)_{L}$ singlets: $S \equiv S_{1}^{+}, T \equiv S_{2}^{+}$and $\mathcal{F} \equiv N$. For $n=1(n=2)$ the multiplets $T$ and $\mathcal{F}$ are $S U(2)_{L}$ triplets (quintuplets) and one has the triplet (quintuplet) model. In all cases, the new multiplets are subject to a discrete symmetry with action $\{T, \mathcal{F}\} \rightarrow\{-T,-\mathcal{F}\}$. This ensures a stable DM candidate, which should be taken as the lightest fermion $\mathcal{F}_{1}^{0} \equiv \mathcal{F}_{\mathrm{DM}} 5$ Detailed analysis of the DM annihilation channels appears in Refs. 6, 17.

With the aforementioned particle content, the Lagrangian contains the following terms:

$$
\mathcal{L} \supset\left\{f_{\alpha \beta} \overline{L_{\alpha}^{c}} L_{\beta} S^{+}+g_{i \alpha} \overline{\mathcal{F}_{i}} T e_{\alpha R}+\text { H.c }\right\}-\frac{1}{2} \overline{\mathcal{F}_{i}^{c}} \mathcal{M}_{i j} \mathcal{F}_{j}-V(H, S, T) .
$$

Here, $L_{\alpha} \sim(1,2,-1)$ are SM lepton doublets, $e_{\alpha R} \sim(1,1,-2)$ are the SM charged lepton singlets and $f_{\alpha \beta}=-f_{\beta \alpha}$ denote Yukawa couplings. Lepton flavors are labeled by lower-case Greek letters, $\alpha, \beta \in\{e, \mu, \tau\}$. The singlet-leptons $e_{\alpha R}$ couple to the exotics $T$ and $\mathcal{F}$ through the Yukawa matrix $g_{i \alpha}$, and the superscript " $c$ " is used to denote charge conjugation. The Lagrangian shows that both $T$ and $\mathcal{F}$ are sequestered from SM neutrinos. We denote the SM Higgs doublet as $H \sim(1,2,1)$.

The combination of the Yukawa terms in Eq. (1) and the term $\sim S^{2}\left(T^{*}\right)^{2}$ in the scalar potential $V(H, S, T)$ (discussed below) explicitly breaks lepton-number symmetry. The models therefore generate radiative neutrino masses, which appear at the three-loop level as shown in Figure 1. Due to the $Z_{2}$ symmetry, the neutral components of the exotic fermions $\mathcal{F}$ do not mix with SM neutrinos at any order in perturbation theory, and similarly there is no mixing between charged leptons and $\mathcal{F}$. In both the triplet and quintuplet models, the charged scalar $S$ can be within reach of $\mathrm{TeV}$ scale collider experiments [6, 7].

\footnotetext{
${ }^{5}$ For $n=0$ the scalar $T$ is charged, while for $n=1$ and $n=2$ the neutral component of the scalar $T$ has non-trivial couplings to the $Z$ boson and id therefore excluded as a DM candidate by direct-detection constraints.
} 


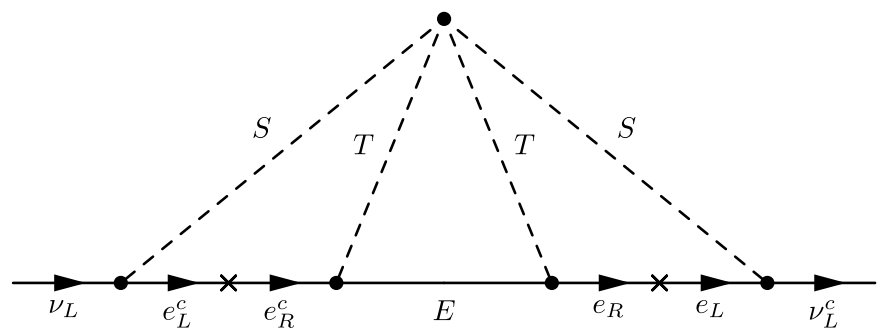

Figure 1: Three-loop diagram for neutrino mass. Here, $S \sim(1,1,2)$ and $T \sim(1,2 n+1,2)$ are beyond-SM scalars while $\mathcal{F} \sim(1,2 n+1,0)$ is a beyond-SM fermion. The case with $n=0$ corresponds to the KNT model [1], while $n=1$ gives the triplet model [6] and $n=2$ gives the quintuplet model [7].

\section{$2.1 \quad$ Triplet Model}

The case with $n=1$ gives the triplet model, for which $\mathcal{F}_{i} \sim(1,3,0)$ and $T \sim(1,3,2)$ are $S U(2)_{L}$ triplets. We write the triplet fields in symmetric-matrix notation as $T=T_{a b}$ and $\mathcal{F}=\mathcal{F}_{a b}$, where $a, b \in\{1,2\}$ are $S U(2)_{L}$ indices. The multiplets contain the following components [6]

$$
\begin{aligned}
& \mathcal{F}_{11}=\mathcal{F}_{L}^{+}, \quad \mathcal{F}_{12}=\mathcal{F}_{21}=\frac{1}{\sqrt{2}} \mathcal{F}_{L}^{0}, \quad \mathcal{F}_{22}=\mathcal{F}_{L}^{-} \equiv\left(\mathcal{F}_{R}^{+}\right)^{c}, \\
& T_{11}=T^{++}, \quad T_{12}=T_{21}=\frac{1}{\sqrt{2}} T^{+},
\end{aligned}
$$

while the triplet mass term gives

$$
-\left(\overline{\mathcal{F}_{i}^{c}}\right)_{a b} \mathcal{M}_{i j}\left(\mathcal{F}_{j}\right)_{c d} \epsilon^{a c} \epsilon^{b d}=-\overline{\mathcal{F}_{i R}^{+}} \mathcal{M}_{i j} \mathcal{F}_{j L}^{+}-\frac{1}{2} \overline{\left(\mathcal{F}_{i L}^{0}\right)^{c}} \mathcal{M}_{i j} \mathcal{F}_{j L}^{0}
$$

The neutral-fermion mass terms are brought to the correct sign by defining the Majorana fermions $\mathcal{F}_{i}^{0}=\mathcal{F}_{i, L}^{0}-\left(\mathcal{F}_{i, L}^{0}\right)^{c}$. Radiative corrections from SM gauge bosons lift the degeneracy between the components of $\mathcal{F}$, leaving $\mathcal{F}^{0}$ as the lightest component [16], though for most purposes this small splitting can be neglected [6]. We work in the diagonal basis with $\mathcal{M}_{i j}=\operatorname{diag}\left(M_{1}, M_{2}, M_{3}\right)$, where $M_{1} \equiv M_{\mathrm{DM}}$ is the lightest triplet-fermion mass. According to the analysis in Ref. [6], the DM mass should lie in the range $2.35-2.75$ $\mathrm{TeV}$, and all triplet members masses should be larger than the DM mass. Accordingly, the triplet scalars are beyond the reach of the LHC, though, as we shall see, they can still be probed indirectly. 
The scalar potential for the triplet model has the form

$$
\begin{aligned}
& V(H, S, T)=-\mu^{2}|H|^{2}+\lambda|H|^{4}+\mu_{S}^{2}|S|^{2}+\frac{\lambda_{S}}{2}|S|^{4}+\mu_{T}^{2}\left[\left(T^{*}\right)^{a b} T_{a b}\right]+\frac{\eta_{1}}{2}\left[\left(T^{*}\right)^{a b} T_{a b}\right]^{2} \\
& +\frac{\eta_{2}}{2}\left(T^{*}\right)^{a b} T_{b c}\left(T^{*}\right)^{c d} T_{d a}+\lambda_{S H}|S|^{2}|H|^{2}+\left\{\bar{\lambda}_{S T}|S|^{2}+\bar{\lambda}_{H T}|H|^{2}\right\}\left[\left(T^{*}\right)^{a b} T_{a b}\right] \\
& -\lambda_{H T}\left(H^{*}\right)^{a} T_{a b}\left(T^{*}\right)^{b c} H_{c}+\frac{\lambda_{S T}}{4}\left(S^{-}\right)^{2} T_{a b} T_{c d} \epsilon^{a c} \epsilon^{b d}+\frac{\lambda_{S T}^{*}}{4}\left(S^{+}\right)^{2}\left(T^{*}\right)^{a b}\left(T^{*}\right)^{c d} \epsilon_{a c} \epsilon_{b d} .
\end{aligned}
$$

Vacuum stability requires that the quantities

$$
\lambda, \lambda_{S}, \eta_{1}+\eta_{2}, \eta_{1}+\frac{1}{2} \eta_{2},\left|\begin{array}{cc}
\lambda & \left(\lambda_{H T}-\bar{\lambda}_{H T}\right)^{0} \\
\left(\lambda_{H T}-\bar{\lambda}_{H T}\right)^{0} & \eta_{1}+\eta_{2}
\end{array}\right|,\left|\begin{array}{cc}
\lambda_{S} & \bar{\lambda}_{S T}^{0} \\
\bar{\lambda}_{S T}^{0} & \eta_{1}+\frac{1}{2} \eta_{2}
\end{array}\right|,
$$

are taken strictly positive, with $\lambda_{\#}^{0}=\min \left(\lambda_{\#}, 0\right)$. Taking the charged scalar squared mass $\mu_{S}^{2}$ and the scalar triplet squared masses as positive ensures the absence of spontaneous charge symmetry breaking and guarantees that $\left\langle T^{0}\right\rangle=0$. The latter is is necessary to preserve the $Z_{2}$ symmetry and retain a stable DM candidate.

\subsection{Quintuplet Model}

The quintuplet model corresponds to $n=2$, in which case one has $\mathcal{F}_{i} \sim(1,5,0)$ and $T \sim(1,5,2)$ as $S U(2)_{L}$ quintuplets. In symmetric-matrix notation, the quintuplets are written as $T_{a b c d}$ and $\mathcal{F}_{a b c d}$, where [7]

$$
\begin{aligned}
& \mathcal{F}_{1111}=\mathcal{F}_{L}^{++}, \mathcal{F}_{1112}=\frac{\mathcal{F}_{L}^{+}}{\sqrt{4}}, \mathcal{F}_{1122}=\frac{\mathcal{F}_{L}^{0}}{\sqrt{6}}, \mathcal{F}_{1222}=\frac{\left(\mathcal{F}_{R}^{+}\right)^{c}}{\sqrt{4}}, \mathcal{F}_{2222}=\left(\mathcal{F}_{R}^{++}\right)^{c}, \\
& T_{1111}=T^{+++}, T_{1112}=\frac{T^{++}}{\sqrt{4}}, T_{1122}=\frac{T^{+}}{\sqrt{6}}, T_{1222}=\frac{T^{0}}{\sqrt{4}}, T_{2222}=T^{-} .
\end{aligned}
$$

Observe that $T^{+}$and $T^{-}$are distinct fields with $T^{-} \neq\left(T^{+}\right)^{*}$. The explicit expansion of the fermion mass term gives

$$
-\frac{1}{2}\left(\overline{\mathcal{F}_{i}^{c}}\right)_{a b c d} \mathcal{M}_{i j}\left(\mathcal{F}_{j}\right)_{e f g h} \epsilon^{a e} \epsilon^{b f} \epsilon^{c g} \epsilon^{d h}+\text { H.c. }=-\overline{\mathcal{F}_{i}^{++}} \mathcal{M}_{i j} \mathcal{F}_{j}^{++}-\overline{\mathcal{F}_{i}^{+}} \mathcal{M}_{i j} \mathcal{F}_{j}^{+}-\frac{1}{2} \overline{\mathcal{F}_{i}^{0}} \mathcal{M}_{i j} \mathcal{F}_{j}^{0},
$$

where $\mathcal{F}^{0}$ is a Majorana fermion and the other four components of $\mathcal{F}$ combine to give two charged (Dirac) fermions:

$$
\mathcal{F}^{++}=\mathcal{F}_{L}^{++}+\mathcal{F}_{R}^{++}, \quad \mathcal{F}^{+}=\mathcal{F}_{L}^{+}-\mathcal{F}_{R}^{+}, \quad \mathcal{F}^{0}=\mathcal{F}_{L}^{0}+\left(\mathcal{F}_{L}^{0}\right)^{c}
$$

Without loss of generality, we again employ the basis with $\mathcal{M}_{i j}=\operatorname{diag}\left(M_{1}, M_{2}, M_{3}\right)$, where $M_{1} \equiv M_{\mathrm{DM}}$. According to the analysis of Ref. [7], the DM mass is expected to lie 
in the range $5.65-6.95 \mathrm{TeV}$, and all quintuplet members should have masses that exceed $M_{\mathrm{DM}} 6$ Consequently the quintuplet scalars cannot be produced directly at the LHC.

The full scalar potential for the quintuplet model that respects the global symmetry $Z_{2}$ is given by

$$
\begin{aligned}
V(H, S, T) & =-\mu^{2}|H|^{2}+\lambda|H|^{4}+\mu_{S}^{2}|S|^{2}+\frac{\lambda_{S}}{2}|S|^{4}+\lambda_{S H}|S|^{2}|H|^{2}+\mu_{T}^{2}\left[\left(T^{*}\right)^{a b c d} T_{a b c d}\right] \\
& +\frac{\eta_{1}}{2}\left[\left(T^{*}\right)^{a b c d} T_{a b c d}\right]^{2}+\frac{\eta_{2}}{2}\left[\left(T^{*}\right)^{a b c d} T_{c d e f}\left(T^{*}\right)^{e f l m} T_{l m a b}\right]+\frac{\eta_{3}}{2}\left[\left(T^{*}\right)^{a b c d} T_{b c d e}\left(T^{*}\right)^{e f l m} T_{a f l m}\right] \\
+ & \left\{\lambda_{S T}|S|^{2}+\lambda_{H T 1}|H|^{2}\right\}\left[\left(T^{*}\right)^{a b c d} T_{a b c d}\right]+\lambda_{H T 2}\left(T^{*}\right)^{a b c d} T_{e b c d}\left(H^{*}\right)^{e} H_{a} \\
& +\frac{\kappa}{4}\left(S^{-}\right)^{2} T_{a b c d} T_{e f g h} \epsilon^{a e} \epsilon^{b f} \epsilon^{c g} \epsilon^{d h}+\text { H.c. }
\end{aligned}
$$

Vacuum stability requires that the following quantities

$$
\begin{gathered}
\lambda, \lambda_{S}, \eta_{1}+\frac{1}{2} \eta_{2}+\frac{1}{2} \eta_{3}, \eta_{1}+\frac{1}{2} \eta_{2}+\frac{5}{8} \eta_{3}, \eta_{1}+\eta_{2}+\eta_{3}>0, \\
\lambda \\
\left|\begin{array}{cc}
\lambda & \left(\lambda_{H T 1}+\frac{3}{4} \lambda_{H T 2}\right)^{0} \\
\left(\lambda_{H T 1}+\frac{3}{4} \lambda_{H T 2}\right)^{0} & \eta_{1}+\frac{1}{2} \eta_{2}+\frac{5}{8} \eta_{3}
\end{array}\right|,\left|\begin{array}{ccc}
\lambda_{S} & \lambda_{S T}^{0} & \lambda_{S T}^{0} \\
\lambda_{S T}^{0} & \eta_{1}+\frac{1}{2} \eta_{2}+\frac{1}{2} \eta_{3} & \left(\eta_{1}+\frac{1}{3} \eta_{2}+\frac{1}{2} \eta_{3}\right)^{0} \\
\lambda_{S T}^{0} & \left(\eta_{1}+\frac{1}{3} \eta_{2}+\frac{1}{2} \eta_{3}\right)^{0} & \eta_{1}+\eta_{2}+\eta_{3}
\end{array}\right|,
\end{gathered}
$$

be strictly positive. Similar to the triplet case, spontaneous charge symmetry breaking is avoided and the neutral quintuplet remains VEV-less by taking the squared masses of the charged scalar and the quintuplet to be positive. This preserves the $Z_{2}$ symmetry.

In the numerical scans performed below, we impose the above mentioned conditions such as vacuum stability, charge non-breaking and $\left\langle T^{0}\right\rangle=0$, and also require the Higgs mass to be within the range reported by ATLAS and CMS, $m_{h}=125.09 \mp 0.21 \mathrm{GeV}$ [17]. We restrict our attention to the perturbativity domain, demanding that the physical vertices in Eqs. (4) and (9) be less than 3. For both the triplet [6] and quintuplet [7] models, we consider the charged singlet scalar mass between $100 \mathrm{GeV}$ and $1 \mathrm{TeV}$. The masses for the scalar multiplet members should be larger than the dark matter mass, i.e., $M_{T}>2.35 \mathrm{TeV}$ and $M_{T}>5.65 \mathrm{TeV}$, for the triplet and quintuplet models, respectively. For the numerical analysis we consider 20,000 sets of benchmark points for both the triplet and quintuplet models. The benchmarks reproduce the observed DM relic density while also achieving viable neutrino masses and avoiding lepton flavor violating constraints (see Refs. [6, 7] for discussion on constraints).

\footnotetext{
${ }^{6}$ The degeneracy between neutral and charged components of $\mathcal{F}$ is again lifted by radiative corrections.
} 


\section{Higgs Mass and Triple Higgs Coupling}

In order to estimate the Higgs mass and the triple Higgs coupling at one-loop, it is necessary to properly define the effective potential, with the Higgs mass being its second derivative and the triple Higgs coupling given by the third derivative:

$$
\begin{aligned}
& m_{h}^{2}=\left.\frac{\partial^{2}}{\partial h^{2}} V_{e f f}^{T=0}(h)\right|_{h=v}, \\
& \lambda_{h h h}=\left.\frac{\partial^{3}}{\partial h^{3}} V_{e f f}^{T=0}(h)\right|_{h=v} .
\end{aligned}
$$

Here $h$ is the real part of the neutral component in the doublet, $v$ is its $\mathrm{VEV}$, and $V_{e f f}^{T=0}(h)$ is the zero temperature one-loop Higgs effective potential. In this work we employ the $\overline{D R}^{\prime}$ scheme, for which the effective potential is given by [18]

$$
V_{e f f}^{T=0}(h)=-\frac{\mu^{2}}{2} h^{2}+\frac{\lambda}{4} h^{4}+\sum_{i} n_{i} \frac{m_{i}^{4}(h)}{64 \pi^{2}}\left(\log \frac{m_{i}^{2}(h)}{\Lambda^{2}}-\frac{3}{2}\right),
$$

where $n_{i}$ is the field multiplicity and $\Lambda$ is the renormalization scale, which we take as the measured value of the Higgs mass, $\Lambda=125.09 \mathrm{GeV}$ [17]. The quantities $m_{i}^{2}(h)$ are the field-dependent squared masses (presented in the appendix for both triplet and quintuplet models). In this class of models, $h$ is the only scalar with a non-zero VEV, so all field-dependent masses can be written as $m_{i}^{2}(h)=\mu_{i}^{2}+\alpha_{i}^{2} h^{2} / 2$, for constant $\alpha_{i}$.

At tree-level, the parameter $\mu^{2}$ in the potential is given by $\mu^{2}=\lambda v^{2}$. After including one-loop corrections, the parameter $\mu^{2}$ is corrected as

$$
\mu^{2}=\lambda v^{2}+\left.\frac{1}{32 \pi^{2}} \sum_{i} n_{i} \alpha_{i} m_{i}^{2}\left(\ln \frac{m_{i}^{2}}{\Lambda^{2}}-1\right)\right|_{h=v, \mu^{2} \equiv \mu^{2}+\delta \mu^{2}},
$$

in order to ensure the one-loop VEV value remains as $v=246 \mathrm{GeV}$. The term $\delta \mu^{2}$ represents the radiative corrections to the $\mu^{2}$-term, due to all fields, and is expected to be dominated by contributions from the new heavy fields. The Higgs mass at one-loop can be similarly defined by

$$
m_{h}^{2}=2 \lambda v^{2}+\frac{v^{2}}{32 \pi^{2}} \sum_{i} n_{i} \alpha_{i}^{2} \log \frac{m_{i}^{2}}{\Lambda^{2}}
$$

where the radiative corrections (i.e., second term in (14)) are also expected to be dominated by contributions from heavy new fields. Although $m_{h}^{2}$ is determined by experimental observations, the doublet quartic coupling $\lambda$ can still be (very) small, relative to the SM value, while reproducing the observed Higgs mass. According to the size and sign of the 
one-loop contribution in (14), the quartic coupling $\lambda$ must be smaller (larger) than the tree-level value $3 m_{h}^{2} / v^{2}$, for a positive (negative) loop contribution.

The triple Higgs coupling is the third derivative of (12), which can be simplified as

$$
\lambda_{h h h}=6 \lambda v+\frac{v}{32 \pi^{2}} \sum_{i} n_{i} \alpha_{i}^{2}\left(\frac{\alpha_{i} v^{2}}{m_{i}^{2}}+3 \log \frac{m_{i}^{2}}{\Lambda^{2}}\right) .
$$

Using (13) and (14), the triple Higgs coupling (15) can be simplified as

$$
\lambda_{h h h}=\frac{3 m_{h}^{2}}{v}\left(1+\frac{v^{4}}{96 \pi^{2} m_{h}^{2}} \sum_{i} \frac{n_{i} \alpha_{i}^{3}}{m_{i}^{2}}\right) .
$$

The size of the radiative effects can be parameterized by the following dimensionless quantities:

$$
\delta \mu^{2}=\frac{\mu^{2}-\lambda v^{2}}{\mu^{2}}, \quad \delta m_{h}^{2}=\frac{m_{h}^{2}-2 \lambda v^{2}}{m_{h}^{2}}, \quad \delta \lambda_{h h h}=\frac{\lambda_{h h h}-6 \lambda v}{\lambda_{h h h}} .
$$

These measure the relative strength of the radiative contributions to the Higgs bare masssquared, the physical mass-squared $\mu^{2}$, and the triple Higgs coupling, respectively. Using the previously mentioned benchmark points, in Fig. 2 we plot the triple Higgs coupling versus the mass-squared parameter $\mu^{2}$, for both triplet and quintuplet models. We also show the relative strength of the radiative contributions to the Higgs mass, triple Higgs coupling and the parameter $\mu^{2}$, as defined in Eq. (17).

One notices from Fig. 2-Top that the mass-squared parameter $\mu^{2}$ can be large, even up to 100 (500) times the Higgs VEV-squared $v^{2}$ for the triplet (quintuplet) models. The larger values are required in order to balance the radiatively induced mass term in the Lagrangian, i.e., the second term on the left-hand side of (13). The radiative corrections can also be negative, depending on the value of the Higgs quartic coupling; i.e., for $\lambda \gtrsim 0.08$. Due to the fact that the extra fields in the quintuplet model are much heavier than those of the triplet model, their radiative contributions are larger and therefore the $\mu^{2}$ parameter values are larger, as it is evident from the figures. From Fig. 2-Bottom, one notices that the relative radiative-contributions to the Higgs mass and triple coupling are proportional, i.e. when the Higgs mass is completely generated radiatively, the triple Higgs coupling is also dominated by radiative effects. We also observe that for most of the benchmark points, in both the triplet and quintuplet models, the mass-squared parameter $\mu^{2}$ is fully radiative, as shown in the palette.

The relevant quantity for collider phenomenology is the relative enhancement in the triple Higgs coupling, with respect to the SM value, which is defined as

$$
\Delta=\frac{\lambda_{h h h}-\lambda_{h h h}^{S M}}{\lambda_{h h h}^{S M}}
$$



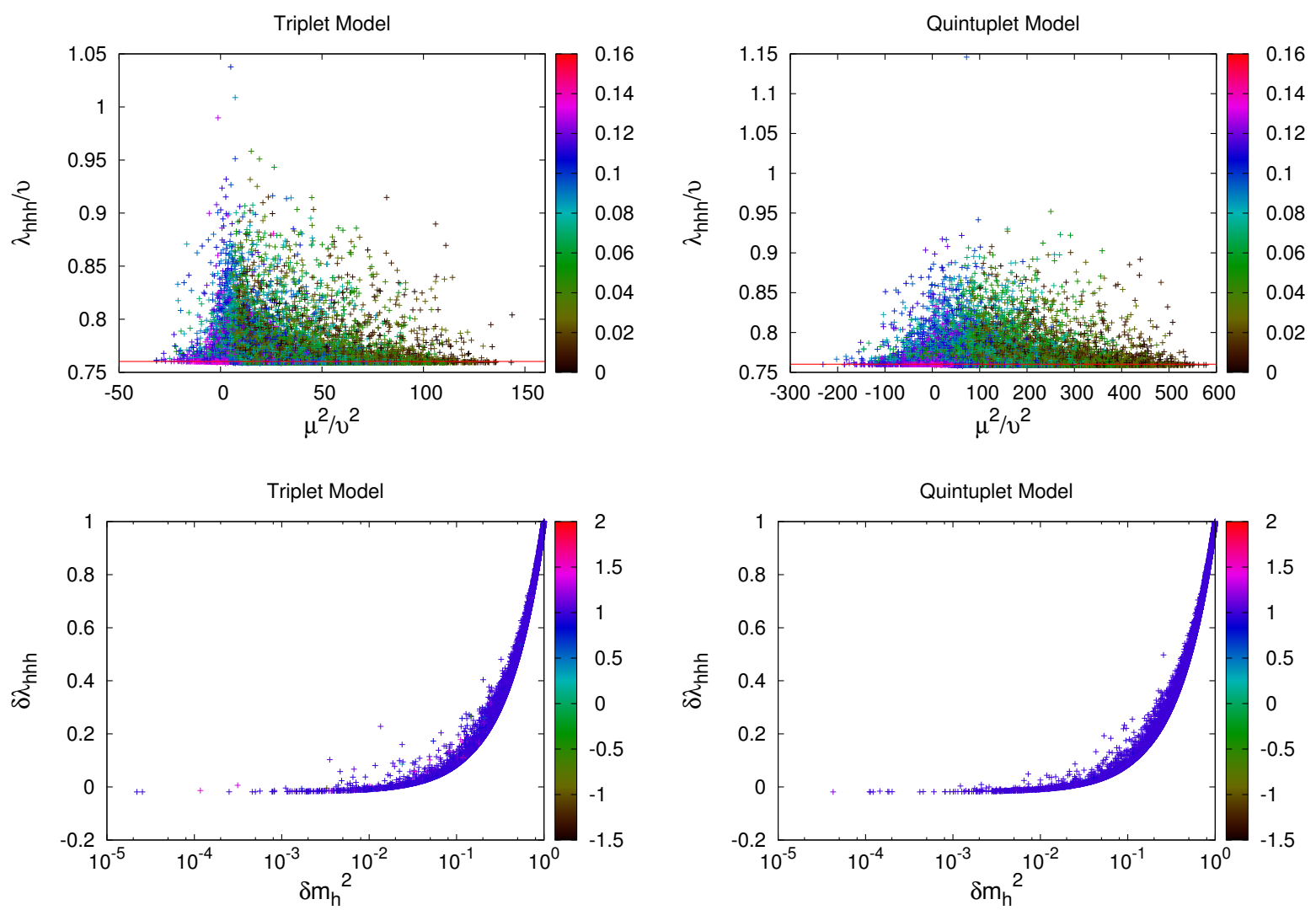

Figure 2: Top: the triple Higgs coupling versus the mass parameter parameter $\mu^{2}$, both in units of the Higgs VEV, for the triplet (left) and quintuplet (right) models. The palette gives the Higgs quartic coupling $\lambda$, and the red line shows the SM triple Higgs coupling value. Bottom: the relative radiative contribution to the triple Higgs coupling versus the relative radiative contribution to the Higgs mass. The palette gives the relative radiative contribution to the Higgs mass parameter $\delta \mu^{2}$.

According to Eq. (16), the relative enhancement of the triple Higgs coupling is given by

$$
\Delta=\frac{\sum_{i \neq S M} \frac{n_{i} \alpha_{i}^{3}}{m_{i}^{2}}}{\frac{96 \pi^{2} m_{h}^{2}}{v^{4}}+\sum_{i=\text { all }} \frac{n_{i} \alpha_{i}^{3}}{m_{i}^{2}}} .
$$

In Fig. 3, we show the relative enhancement of the triple Higgs coupling, Eq. (19), for the benchmark sets used previously. The figure shows that the relative enhancement of the triple Higgs coupling, with respect to the SM, are larger for large values of the quartic coupling $\lambda$, and smaller for small values of the charged scalar mass. Also, one notices that the relative enhancement in the triple Higgs coupling, with respect to the SM value, is always positive, contrary to other models [19, 20], and furthermore it can exceed $35 \%$ for 

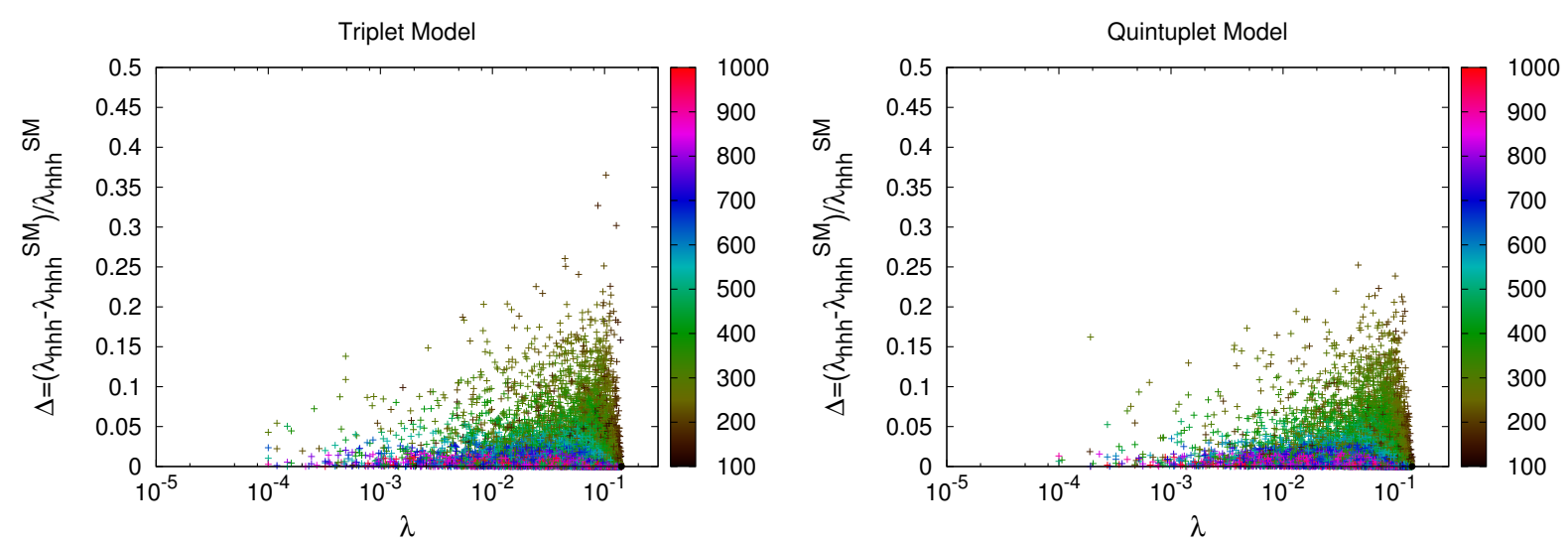

Figure 3: The relative enhancement in the triple Higgs coupling with respect to the SM, $\Delta$, versus the Higgs quartic coupling. The palette shows the mass of the charged scalar $S^{+}$in $\mathrm{GeV}$. The black point at $\left(\lambda=\lambda^{S M}, 0\right)$ refers to the $S M$.

both the triplet and quintuplet models.

\section{Electroweak Phase Transition}

The SM cannot successfully explain baryogenesis [21] for two reasons: (1) the CP violating source in the CKM matrix is too small and (2) the electroweak phase transition (EWPT) is not strongly first order. The latter is required to suppress the $\mathrm{B}+\mathrm{L}$ violating processes in the broken phase, inside the bubble, when its wall is expanding during the transition. In the SM, the criterion for a strongly first-order EWPT [22],

$$
v\left(T_{c}\right) / T_{c}>1
$$

is not fulfilled since the ratio is given by $v_{c} / T_{c} \sim \lambda$, which would require a Higgs mass below $42 \mathrm{GeV}$ [23]. Here $T_{c}$ is the critical temperature at which the effective potential exhibits two degenerate minima, one at zero and the other at $v\left(T_{c}\right)$. Both $T_{c}$ and $v\left(T_{c}\right)$ are determined using the full effective potential at finite temperature, which is given by [24]

$$
\begin{aligned}
V_{e f f}(h, T) & =V_{e f f}^{T=0}(h)+\frac{T^{4}}{2 \pi^{2}} \sum_{i} n_{i} J_{B, F}\left(m_{i}^{2} / T^{2}\right)+V_{\text {ring }}(h, T) ; \\
J_{B, F}(\alpha) & =\int_{0}^{\infty} x^{2} \log \left(1 \mp \exp \left(-\sqrt{x^{2}+\alpha}\right)\right) .
\end{aligned}
$$

In the above, we include an important leading term from the higher-order loop corrections, which can play an important role during the EWPT dynamics, namely the so-called daisy 
contributions [25]

$$
V_{\text {ring }}(h, T)=-\frac{T}{12 \pi} \sum_{i} n_{i}\left\{\tilde{m}_{i}^{3}(h, T)-m_{i}^{3}(h)\right\}
$$

The summation is over scalar and longitudinal gauge degrees of freedom, with $\tilde{m}_{i}^{2}(h, T)=$ $m_{i}^{2}(h)+\Pi(T)$ their thermal masses, and $\Pi(T)$ are the thermal parts of the self energy (given in the appendix). For our analysis we include the daisy contributions by following an alternative approach to Eq. (23), i.e. by replacing the field dependent masses of the scalar and longitudinal gauge fields by their thermal masses $\tilde{m}_{i}^{2}(h, T)$ in the full effective potential (21). In order to account for all the (heavy and light) degrees of freedom, we evaluate the integrals (22) numerically.

The strength of the EWPT can be improved when new bosonic degrees of freedom are present, as occurs in the present models. It is clear from (14) that for large values for the couplings $\left\{\lambda_{S H}, \lambda_{H T}, \bar{\lambda}_{H T}\right.$ for triplet model and $\lambda_{S H}, \lambda_{H T 1,2}$ for quintuplet model $\}$ and/or small mass-values for the extra (singlet and multiplet) scalars, the one-loop correction to the Higgs mass can be significant, allowing the Higgs self-coupling to be smaller. Consequently one can fulfill the criterion (20) without conflicting with recent Higgs mass measurements [17].

Analyses of similar models [4, 19, 26] has shown that extra scalars can help to generate a strongly first order EWPT by: (a) relaxing the Higgs self-coupling $\lambda$ to be as small as $\mathcal{O}\left(10^{-4}\right)$; and (b) enhancing the value of the effective potential at the wrong vacuum at the critical temperature, without suppressing the ratio $v\left(T_{c}\right) / T_{c}$, which relaxes the severe bound on the mass of the SM Higgs.
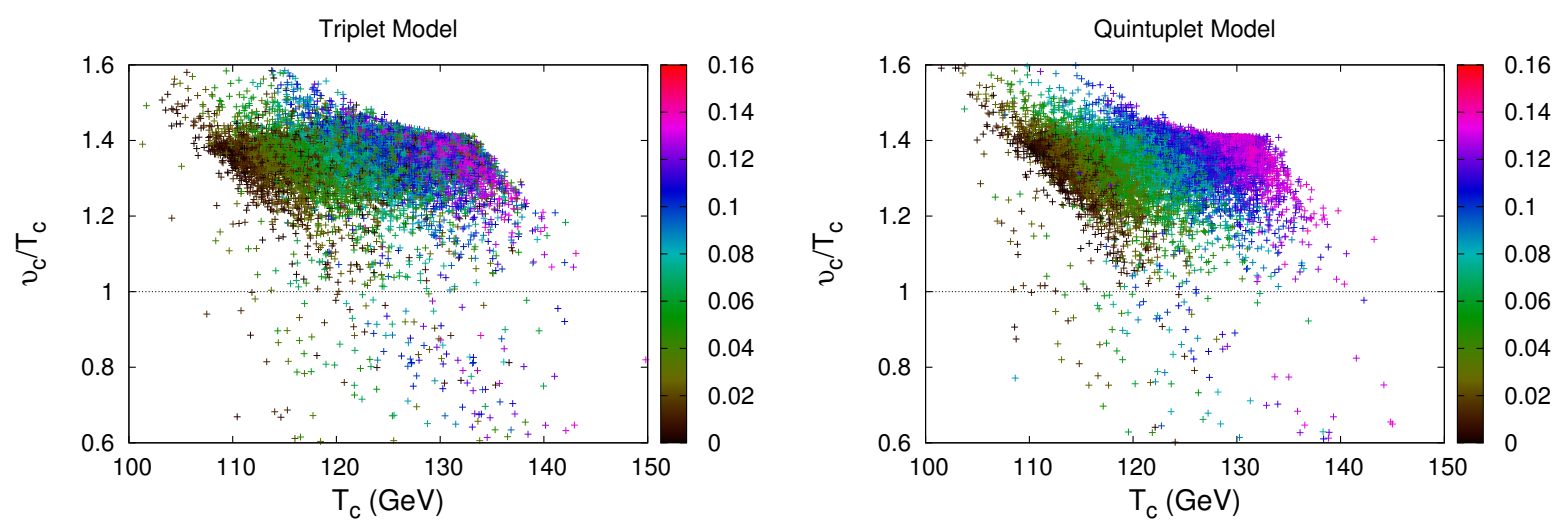

Figure 4: The ratio $v\left(T_{c}\right) / T_{c}$ versus the critical temperature for the (left) triplet and (right) quintuplet models. The palette shows the Higgs quartic coupling $\lambda$. 
In Fig. 4, we plot the ratio $v\left(T_{c}\right) / T_{c}$ verses the critical temperature $T_{c}$, using the 20,000 benchmark points for the triplet and quintuplet models. The figure shows that the EWPT is strongly first-order for a majority of the benchmark sets, with the ratio $v\left(T_{c}\right) / T_{c}$ predominantly taking values between 1.2 and 1.5 in both the triplet and quintuplet models. The transition temperature is a bit larger than the typical SM value $\sim 100 \mathrm{GeV}$, and can be as large as $150 \mathrm{GeV}$ and $140 \mathrm{GeV}$ for the triplet and quintuplet models, respectively. One can read from the palettes in Fig. 4 that, for fixed Higgs quartic coupling values, the ratio $v\left(T_{c}\right) / T_{c}$ is inversely proportional to the critical temperature.

Inspecting Fig. 4, one is lead to the conclusion that the increased EWPT strength is not only a consequence of a small Higgs quartic coupling $\lambda$, but can also be due to the transition dynamics; the existence of heavy scalars makes the Higgs VEV slowly decaying with respect to the temperature. Consequently the evolving (increasing or decreasing) effective potential at the wrong vacuum makes the transition occurring at the mentioned temperature values, therefore giving a large ratio $v\left(T_{c}\right) / T_{c}$.

An interesting issue, discussed in the literature [27], is a possible correlation between the EWPT strength and the relative enhancement in the triple Higgs coupling $\Delta$. In Ref. [19] it was shown that such a correlation is not clear for a model with extra charged and neutral scalars from two inert doublets. In Fig. 5, we plot the relative enhancement of the triple Higgs coupling, $\Delta$, versus the ratio $v\left(T_{c}\right) / T_{c}$, for the 20,000 benchmarks for the triplet and quintuplet models. From Figure 5, it is not clear whether a correlation between the relative enhancement in the triple Higgs coupling and the EWPT strength exists. This issue deserves a detailed and model-independent investigation.

\section{$5 \quad$ The Higgs decay channels $h \rightarrow \gamma \gamma$ and $h \rightarrow \gamma Z$}

In July 2012, the ATLAS [28] and CMS [29] collaborations announced the observation of a scalar particle with mass $\simeq 125 \mathrm{GeV}$, with roughly $5 \sigma$ confidence level. Subsequently this value was updated to $m_{h}=125.09 \pm 0.21 \mathrm{GeV}$ [17]. An important question is whether or not this really is the SM Higgs or an alternative Higgs-like state with different properties. Indeed, a fit to the data, performed by both the ATLAS and CMS collaborations, seems to (almost) show agreement with the SM, with the reported values being $1.17 \mp 0.27$ [30] and $1.13 \pm 0.24$ [31], respectively.

Defining $R_{\gamma \gamma}$ as the branching ratio of $h \rightarrow \gamma \gamma$ scaled by the SM value, we find that 


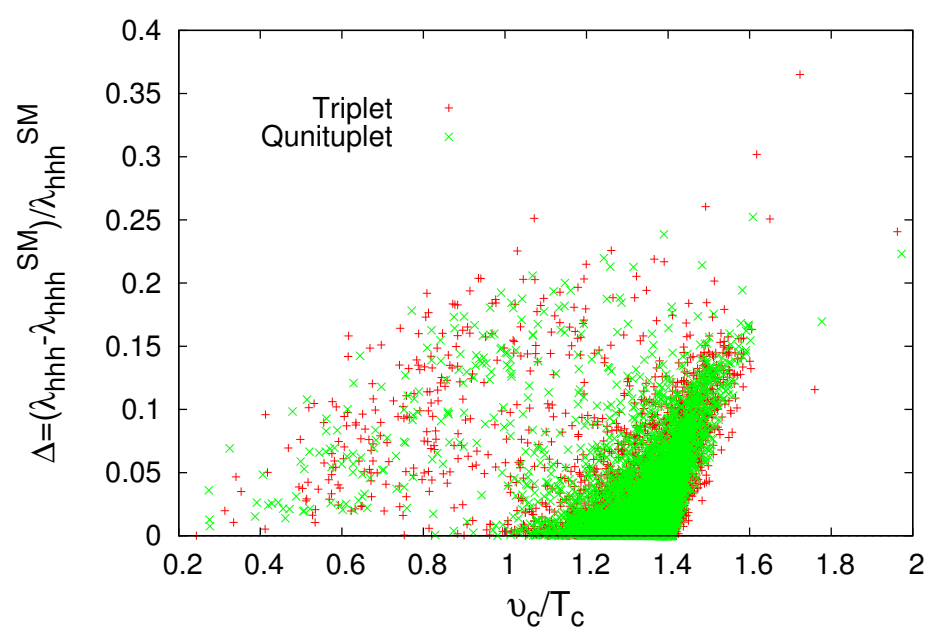

Figure 5: The relative enhancement in the triple Higgs coupling $\Delta$ versus the ratio $v\left(T_{c}\right) / T_{c}$ for the benchmark points used previously.

the present models give

$$
R_{\gamma \gamma}=\frac{\mathcal{B}(h \rightarrow \gamma \gamma)}{\mathcal{B}^{S M}(h \rightarrow \gamma \gamma)}=\left|1+\frac{v^{2}}{2} \frac{\sum_{i} \frac{\vartheta_{i}}{m_{X_{i}}^{2}} A_{0}^{\gamma \gamma}\left(\tau_{i}\right)}{A_{1}^{\gamma \gamma}\left(\tau_{W}\right)+N_{c} Q_{t}^{2} A_{1 / 2}^{\gamma \gamma}\left(\tau_{t}\right)}\right|^{2},
$$

where $i$ stands for all charged scalars $X_{i}, \tau_{i}=m_{h}^{2} / 4 m_{X_{i}}^{2}$, with $m_{X_{i}}$ being the mass of the charged particle $X_{i}$ running inside the loop, $N_{c}=3$ is the color number, and $Q_{t}$ is the top quark electric charge in units of $|e|$. The parameters $\vartheta_{i}$ are given for triplet and quintuplet members in Table 1. In the above, the loop amplitudes $A_{k}^{\gamma \gamma}$ for spin 0, spin $1 / 2$ and spin 1 particles are given by 32

$$
\begin{aligned}
A_{0}^{\gamma \gamma}(x) & =-x^{-2}[x-f(x)], \\
A_{1 / 2}^{\gamma \gamma}(x) & =2 x^{-2}[x+(x-1) f(x)], \\
A_{1}^{\gamma \gamma}(x) & =-x^{-2}\left[2 x^{2}+3 x+3(2 x-1) f(x)\right], \\
f(x) & =\left\{\begin{array}{cc}
\arcsin ^{2}(\sqrt{x}) & x \leq 1 \\
-\frac{1}{4}\left[\log \frac{1+\sqrt{1-x^{-1}}}{1-\sqrt{1-x^{-1}}}-i \pi\right]^{2} & x>1 .
\end{array}\right.
\end{aligned}
$$

Another important Higgs decay channel that can be modified by the presence of extra charged scalars is $h \rightarrow \gamma Z$. This channel is similarly parameterized as

$$
R_{\gamma Z}=\frac{\mathcal{B}(h \rightarrow \gamma Z)}{\mathcal{B}^{S M}(h \rightarrow \gamma Z)}=\left|1+s_{\mathrm{w}} c_{\mathrm{w}} v^{2} \frac{\sum_{i} \frac{\varkappa_{i}}{m_{X_{i}}^{2}} A_{0}^{\gamma Z}\left(\tau_{i}, \zeta_{i}\right)}{c_{\mathrm{w}}^{2} A_{1}^{\gamma Z}\left(\tau_{W}, \zeta_{W}\right)+2\left(1-8 s_{\mathrm{w}}^{2} / 3\right) A_{1 / 2}^{\gamma Z}\left(\tau_{t}, \zeta_{t}\right)}\right|^{2}
$$




\begin{tabular}{|c|c|c|c|}
\hline Model & Charged fields & $\vartheta_{i}$ & $\varkappa_{i}$ \\
\hline \hline \multirow{4}{*}{ Triplet } & $S^{+}$ & $\lambda_{S H}$ & $\frac{s_{w}}{c_{w}} \lambda_{S H}$ \\
\cline { 2 - 4 } & $T^{+}$ & $\frac{2 \lambda_{H T}-\bar{\lambda}_{H T}}{2}$ & $\frac{s_{w}}{c_{w}} \frac{2 \lambda_{H T}-\bar{\lambda}_{H T}}{2}$ \\
\cline { 2 - 4 } & $T^{++}$ & $4 \lambda_{H T}$ & $-\frac{2-4 s_{w}^{2}}{s_{w} c_{w}} \lambda_{H T}$ \\
\hline \hline \multirow{5}{*}{ Quintuplet } & $S^{+}$ & $\lambda_{S H}$ & $\frac{s_{w}}{c_{w}} \lambda_{S H}$ \\
\cline { 2 - 4 } & $T^{-}$ & $\lambda_{H T 1}+\lambda_{H T 2}$ & $\frac{2+s_{w}^{2}}{s_{w} c_{w}}\left(\lambda_{H T 1}+\lambda_{H T 2}\right)$ \\
\cline { 2 - 4 } & $T^{+}$ & $\frac{2 \lambda_{H T 1}+\lambda_{H T 2}}{2}$ & $\frac{s_{w}}{c_{w}} \frac{2 \lambda_{H T 1}+\lambda_{H T 2}}{2}$ \\
\cline { 2 - 4 } & $T^{++}$ & $4 \lambda_{H T 1}+\lambda_{H T 2}$ & $-\frac{1-2 s_{w}^{2}}{s_{w} c_{w}} \frac{4 \lambda_{H T 1}+\lambda_{H T 2}}{2}$ \\
\cline { 2 - 4 } & $T^{+++}$ & $9 \lambda_{H T 1}$ & $-\frac{6-9 s_{w}^{2}}{s_{w} c_{w}} \lambda_{H T 1}$ \\
\hline
\end{tabular}

Table 1: The parameters $\vartheta_{i}$ and $\varkappa_{i}$, which are relevant for the Higgs decay channels $h \rightarrow \gamma \gamma$ and $h \rightarrow \gamma Z$.

where $\zeta_{i}=m_{Z}^{2} / 4 m_{X_{i}}^{2}$, and the functions $A_{k}^{\gamma Z}$ are given by 32

$$
\begin{aligned}
& A_{0}^{\gamma Z}(x, y)=I_{1}(x, y), \\
& A_{1 / 2}^{\gamma Z}(x, y)=I_{1}(x, y)-I_{2}(x, y), \\
& A_{1}^{\gamma Z}(x, y)=\left[(1+2 x) s_{\mathrm{w}}^{2} / c_{\mathrm{w}}^{2}-(5+2 x)\right] I_{1}(x, y)+4\left(3-s_{\mathrm{w}}^{2} / c_{\mathrm{w}}^{2}\right) I_{2}(x, y) \text {, } \\
& I_{1}(x, y)=-\frac{1}{2(x-y)}+\frac{f(x)-f(y)}{2(x-y)^{2}}+\frac{y[g(x)-g(y)]}{(x-y)^{2}}, I_{2}(x, y)=\frac{f(x)-f(y)}{2(x-y)} \text {, } \\
& g(x)=\left\{\begin{array}{cc}
\sqrt{x^{-1}-1} \arcsin (\sqrt{x}) & x \leq 1 \\
\frac{\sqrt{1-x^{-1}}}{2}\left[\log \frac{1+\sqrt{1-x^{-1}}}{1-\sqrt{1-x^{-1}}}-i \pi\right] & x>1 .
\end{array}\right.
\end{aligned}
$$

The parameters $\varkappa_{i}$ are shown in Table 1 for both the triplet and quintuplet models.

The deviation of the channels $h \rightarrow \gamma \gamma, \gamma Z$ from their SM values is sensitive to the mass of the scalars and the strength with which they couple to the Higgs doublet, i.e. on the parameters $m_{S}^{2}, m_{+}^{2}, m_{++}^{2}, \lambda_{S H}, \lambda_{H T}$ and $\bar{\lambda}_{H T}$ for the triplet model, and on $m_{S}^{2}, m_{+}^{2}$, $m_{++}^{2}, m_{+++}^{2}, \lambda_{S H}$ and $\lambda_{H T 1,2}$ for the quintuplet model. Depending on the relative sign of the couplings to the Higgs doublet, the new contributions can strengthen or weaken the deviation of $\mathcal{B}(h \rightarrow \gamma \gamma)$ from its SM value. In Fig. 6, we present $R_{\gamma \gamma}$ versus $R_{\gamma Z}$ for the considered 20,000 sets of benchmark parameters for both the triplet and quintuplet models.

We remark that some benchmarks in the triplet model are already excluded by the recent measurements of ATLAS [17] and CMS [31, while more precise measurements are required to probe benchmarks for the quintuplet model. In contrast to other models with extra singlets [4] and doublets [19], the decay channel $h \rightarrow \gamma Z$ can be significantly 


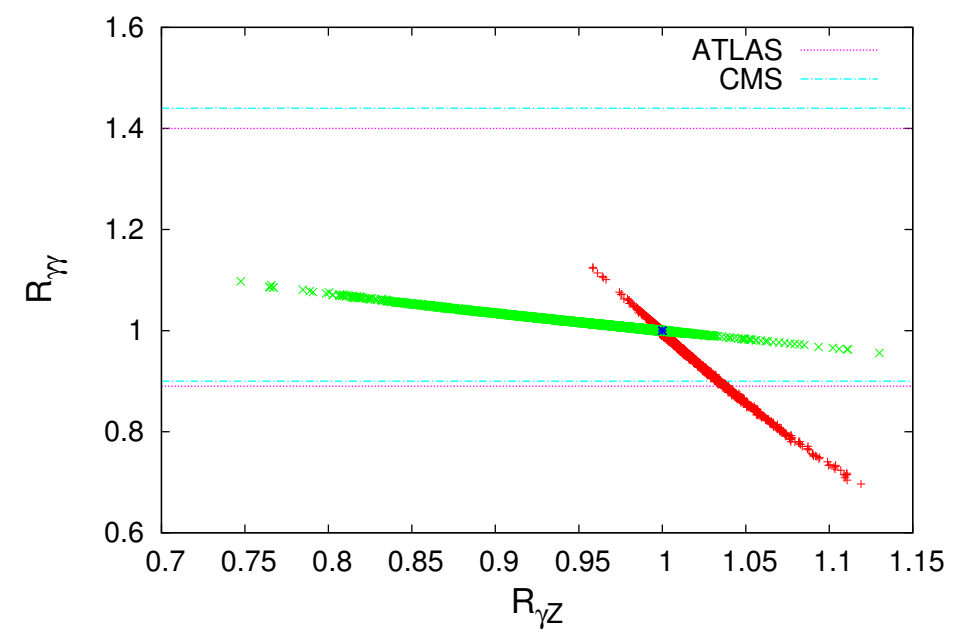

Figure 6: The modified Higgs decay rates $B(h \rightarrow \gamma \gamma)$ vs $B(h \rightarrow \gamma Z)$, scaled by their $S M$ values, due to the extra charged scalars, for 20,000 randomly chosen sets of benchmark parameters for the triplet (red) and quintuplet (green) models. The intervals between the magenta (green) lines represent the ATLAS (CMS) recent measurements on the $h \rightarrow \gamma \gamma$ channel, and the blue point represents the SM.

modified, with respect to the SM value, particularly in the quintuplet model. This can be understood from the large $\varkappa_{i}$ coupling values for the scalar multiplet members in Table 1 ,

\section{Conclusion}

Models of radiative neutrino mass with DM candidates can explain some of the shortcomings of the SM while generating observable experimental signals. In this work, we performed a detailed study of the scalar-sector phenomenology for a pair of three-loop neutrino mass models with DM candidates. The models, referred to as the triplet [6] and quintuplet [7] models, generate neutrino mass via a diagram with the same topology as the KNT model. We investigated the effect of the extra scalars on the Higgs mass, the triple Higgs coupling, and the Higgs decay channels $h \rightarrow \gamma \gamma, \gamma Z$. We also studied the strength of the electroweak phase transition. In both models, it was shown that the beyond-SM multiplets can modify the triple Higgs coupling and the Higgs decay channels away from their SM values. The electroweak phase transition was found to be strongly first-order in significant regions of parameter space. Measurements of the Higgs decay channels already exclude some regions of parameter space for the triplet model, and future improvements will further explore the parameter space for both models. 


\section{Acknowledgments}

AA thanks the ICTP for the hospitality during the last stage of this work. AA is supported by the Algerian Ministry of Higher Education and Scientific Research under the CNEPRU Project No D01720130042. KM is supported by the Australian Research Council.

\section{A Field Dependent Masses}

The charged scalar and SM field-dependent masses are given by:

$$
\begin{aligned}
m_{\chi}^{2} & =-\mu^{2}+\lambda h^{2}+\Pi_{H}, m_{h}^{2}=-\mu^{2}+3 \lambda h^{2}+\Pi_{H}, m_{t}^{2}=\frac{y_{t}^{2}}{2} h^{2}, m_{W}^{2}=m_{W_{3} W_{3}}^{2}=\frac{g_{2}^{2}}{4} h^{2}+\Pi_{W}, \\
m_{B B}^{2} & =\frac{g_{1}^{2}}{4} h^{2}+\Pi_{B}, m_{W_{3} B}^{2}=\frac{g_{2} g_{1}}{4} h^{2}, m_{S}^{2}=\mu_{S}^{2}+\frac{\lambda_{S H}}{2} h^{2}+\Pi_{S},
\end{aligned}
$$

with

$$
\begin{aligned}
& \Pi_{H}=\left(12 \lambda+9 g^{2}+3 g^{\prime 2}+3 y_{t}^{2}+2 \lambda_{S H}\right) \frac{T^{2}}{12}, \Pi_{S}=\left(4 \lambda_{S H}+4 \lambda_{S}+3 g^{\prime 2}\right) \frac{T^{2}}{12} \\
& \Pi_{W}^{L}=\Pi_{W_{3}}^{L}=\frac{11}{6} g^{2} T^{2}, \Pi_{B}^{L}=\frac{27}{16} g^{\prime 2} T^{2}, \Pi_{W}^{T}=\Pi_{B}^{T}=0 .
\end{aligned}
$$

Here, we ignored the triplet and quintuplet contributions since they decouple from the thermal plasma due to their large masses, relative to the relevant typical temperature of $\mathcal{O}(100 \mathrm{GeV})$.

The triplet members field dependant masses are given by:

$$
\begin{aligned}
m_{0}^{2} & =\mu_{T}^{2}+\frac{\lambda_{H T}-\bar{\lambda}_{H T}}{2} h^{2}+\Pi_{T}, m_{+}^{2}=\mu_{T}^{2}+\frac{2 \lambda_{H T}-\bar{\lambda}_{H T}}{4} h^{2}+\Pi_{T}, \\
m_{++}^{2} & =\mu_{T}^{2}+\frac{\lambda_{H T}}{2} h^{2}+\Pi_{T}, \Pi_{T}=\left(9 g^{2}+3 g^{\prime 2}+2 \bar{\lambda}_{H T}+4 \lambda_{H T}+2 \bar{\lambda}_{S T}\right) \frac{T^{2}}{12},
\end{aligned}
$$

and the quintuplet members field dependant masses are given by:

$$
\begin{aligned}
m_{-}^{2} & =\mu_{T}^{2}+\frac{\lambda_{H T 1}+\lambda_{H T 2}}{2} h^{2}+\Pi_{T}, m_{0}^{2}=\mu_{T}^{2}+\frac{4 \lambda_{H T 1}+3 \lambda_{H T 2}}{8} h^{2}+\Pi_{T}, \\
m_{+}^{2} & =\mu_{T}^{2}+\frac{2 \lambda_{H T 1}+\lambda_{H T 2}}{4} h^{2}+\Pi_{T}, m_{++}^{2}=\mu_{T}^{2}+\frac{4 \lambda_{H T 1}+\lambda_{H T 2}}{8} h^{2}+\Pi_{T}, \\
m_{+++}^{2} & =\mu_{T}^{2}+\frac{\lambda_{H T 1}}{2} h^{2}+\Pi_{T}, \Pi_{T}=\left(9 g^{2}+3 g^{\prime 2}+2 \lambda_{S T}+4 \lambda_{H T 1}+2 \lambda_{H T 2}\right) \frac{T^{2}}{12} .
\end{aligned}
$$

\section{References}

[1] L. M. Krauss, S. Nasri and M. Trodden, Phys. Rev. D 67, 085002 (2003) hep-ph/0210389. 
[2] E. A. Baltz and L. Bergstrom, Phys. Rev. D 67, 043516 (2003) hep-ph/0211325].

[3] K. Cheung and O. Seto, Phys. Rev. D 69, 113009 (2004) hep-ph/0403003.

[4] A. Ahriche and S. Nasri, JCAP 1307, 035 (2013) [arXiv:1304.2055].

[5] A. Ahriche, S. Nasri and R. Soualah, arXiv:1403.5694 [hep-ph].

[6] A. Ahriche, C. S. Chen, K. L. McDonald and S. Nasri, Phys. Rev. D 90, 015024 (2014) [arXiv:1404.2696 [hep-ph]].

[7] A. Ahriche, K. L. McDonald and S. Nasri, JHEP 1410 (2014) 167 arXiv:1404.5917 [hep-ph]].

[8] C. S. Chen, K. L. McDonald and S. Nasri, Phys. Lett. B 734, 388 (2014) arXiv:1404.6033 [hep-ph]].

[9] A. Ahriche, K. L. McDonald, S. Nasri and T. Toma, Phys. Lett. B 746, 430 (2015) arXiv:1504.05755 [hep-ph]].

[10] A. Ahriche, K. L. McDonald and S. Nasri, arXiv:1508.02607 [hep-ph].

[11] E. Ma, Phys. Rev. D 73, 077301 (2006) hep-ph/0601225; M. Aoki, S. Kanemura and O. Seto, Phys. Rev. Lett. 102, 051805 (2009) [arXiv:0807.0361 [hep-ph]]; M. Aoki, S. Kanemura and O. Seto, Phys. Rev. D 80, 033007 (2009) arXiv:0904.3829 [hepph]]; M. Aoki, S. Kanemura, T. Shindou and K. Yagyu, JHEP 1007, 084 (2010) [JHEP 1011, 049 (2010)] arXiv:1005.5159 [hep-ph]]; S. Kanemura, O. Seto and T. Shimomura, Phys. Rev. D 84, 016004 (2011) [arXiv:1101.5713 [hep-ph]]; M. Aoki, S. Kanemura and K. Yagyu, Phys. Lett. B 702, 355 (2011) [Erratum-ibid. B 706, 495 (2012)] arXiv:1105.2075 [hep-ph]]; M. Lindner, D. Schmidt and T. Schwetz, Phys. Lett. B 705, 324 (2011) arXiv:1105.4626 [hep-ph]]; S. Kanemura, T. Nabeshima and H. Sugiyama, Phys. Rev. D 85, 033004 (2012) [arXiv:1111.0599 [hep-ph]].

[12] Y. H. Ahn and H. Okada, Phys. Rev. D 85, 073010 (2012) arXiv:1201.4436 [hep-ph]]; S. S. C. Law and K. L. McDonald, Phys. Lett. B 713, 490 (2012) arXiv:1204.2529 [hep-ph]]; R. Bouchand and A. Merle, JHEP 1207, 084 (2012) [arXiv:1205.0008 [hepph]]. G. Guo, X. -G. He and G. -N. Li, JHEP 1210, 044 (2012) arXiv:1207.6308 [hep-ph]]; P. S. Bhupal Dev and A. Pilaftsis, Phys. Rev. D 87, 053007 (2013) arXiv:1212.3808 [hep-ph]]; M. Gustafsson, J. M. No and M. A. Rivera, Phys. Rev. Lett. 110, no. 21, 211802 (2013) arXiv:1212.4806 [hep-ph]]. 
[13] M. Aoki, J. Kubo and H. Takano, Phys. Rev. D 87, 116001 (2013) arXiv:1302.3936 [hep-ph]]. Y. Kajiyama, H. Okada and K. Yagyu, Nucl. Phys. B 874, 198 (2013) arXiv:1303.3463 [hep-ph]]; Y. Kajiyama, H. Okada and T. Toma, Phys. Rev. D 88, no. 1, 015029 (2013) arXiv:1303.7356; S. S. C. Law and K. L. McDonald, JHEP 1309, 092 (2013) arXiv:1305.6467 [hep-ph]]; D. Restrepo, O. Zapata and C. E. Yaguna, JHEP 1311, 011 (2013) [arXiv:1308.3655 [hep-ph]]; E. Ma, I. Picek and B. Radovcic Phys. Lett. B 726, 744 (2013) [arXiv:1308.5313 [hep-ph]]; V. Brdar, I. Picek and B. Radovcic, Phys. Lett. B 728, 198 (2014) [arXiv:1310.3183 [hep-ph]]; H. Okada and K. Yagyu, Phys. Rev. D 89, no. 5, 053008 (2014) arXiv:1311.4360 [hep-ph]]; S. Baek, H. Okada and T. Toma, arXiv:1312.3761 [hep-ph]; S. Baek, H. Okada and T. Toma, arXiv:1401.6921 [hep-ph]; H. Okada, arXiv:1404.0280 [hep-ph]; J. N. Ng and A. de la Puente, Phys. Rev. D 90, no. 9, 095018 (2014) arXiv:1404.1415 [hep-ph]].

[14] S. Kanemura, T. Matsui and H. Sugiyama, Phys. Rev. D 90, 013001 (2014) arXiv:1405.1935 [hep-ph]]; H. Okada and K. Yagyu, Phys. Rev. D 90, 035019 (2014) arXiv:1405.2368 [hep-ph]]; S. Kanemura, N. Machida and T. Shindou, Phys. Lett. B 738, 178 (2014) arXiv:1405.5834 [hep-ph]]; M. Aoki and T. Toma, JCAP 1409, 016 (2014) [arXiv:1405.5870 [hep-ph]]; H. Ishida and H. Okada, arXiv:1406.5808 [hep-ph]; H. Okada and Y. Orikasa, Phys. Rev. D 90, 075023 (2014) [arXiv:1407.2543 [hep-ph]]; H. Okada, T. Toma and K. Yagyu, Phys. Rev. D 90, 095005 (2014) arXiv:1408.0961 [hep-ph]]; H. Hatanaka, K. Nishiwaki, H. Okada and Y. Orikasa, arXiv:1412.8664 [hep-ph];

[15] S. Baek, H. Okada and K. Yagyu, arXiv:1501.01530 [hep-ph]; L. G. Jin, R. Tang and F. Zhang, Phys. Lett. B 741, 163 (2015) arXiv:1501.02020 [hep-ph]]; H. Okada, arXiv:1503.04557 [hep-ph]; H. Okada, N. Okada and Y. Orikasa, arXiv:1504.01204 [hep-ph]; P. Culjak, K. Kumericki and I. Picek, Phys. Lett. B 744, 237 (2015) arXiv:1502.07887 [hep-ph]]; D. Restrepo, A. Rivera, M. Sanchez-Pelaez, O. Zapata and W. Tangarife, arXiv:1504.07892 [hep-ph]; S. Kashiwase, H. Okada, Y. Orikasa and T. Toma, arXiv:1505.04665 [hep-ph]; T. A. Chowdhury and S. Nasri, arXiv:1506.00261 [hep-ph]; M. Aoki, T. Toma and A. Vicente, arXiv:1507.01591 [hep-ph]; K. Nishiwaki, H. Okada and Y. Orikasa, arXiv:1507.02412 [hep-ph]. W. Wang and Z. L. Han, arXiv:1508.00706 [hep-ph]; A. Aranda and E. Peinado, arXiv:1508.01200 [hep-ph]. 
[16] M. Cirelli and A. Strumia, New J. Phys. 11, 105005 (2009) arXiv:0903.3381 [hep$\mathrm{ph}]$.

[17] G. Aad et al. [ATLAS and CMS Collaborations], Phys. Rev. Lett. 114 (2015) 191803 arXiv:1503.07589 [hep-ex]].

[18] S.P. Martin, Phys. Rev. D 65, 116003 (2002) [hep-ph/0111209].

[19] A. Ahriche, G. Faisel, S. Y. Ho, S. Nasri and J. Tandean, arXiv:1501.06605 [hep-ph].

[20] A. Ahriche, A. Arhrib and S. Nasri, JHEP 1402 (2014) 042 arXiv:1309.5615 [hep$\mathrm{ph}]$.

[21] V.A. Kuzmin, V.A. Rubakov and M.E. Shaposhnikov, Phys. Lett. B155, 36-42 (1985).

[22] M.E. Shaposhnikov, Nucl. Phys. B 287, 757-775 (1987); B 299, 797-817 (1988).

[23] A.I. Bochkarev and M.E. Shaposhnikov, Mod. Phys. Lett A2, 417-427 (1987).

[24] L. Dolan and R. Jackiw, Phys. Rev. D 9, 3320-3341 (1974); S. Weinberg, Phys. Rev. D 9, 3357-3378 (1974).

[25] M.E. Carrington, Phys. Rev. D45, 2933-2944 (1992).

[26] A. Ahriche, Phys. Rev. D 75, 083522 (2007) hep-ph/0701192]; A. Ahriche and S. Nasri, Phys. Rev. D 83, 045032 (2011) arXiv:1008.3106 [hep-ph]]; A. Ahriche and S. Nasri, Phys. Rev. D 85, 093007 (2012) arXiv:1201.4614 [hep-ph]].

[27] S. Kanemura, Y. Okada and E. Senaha, Phys. Lett. B 606 (2005) 361 [hep-ph/0411354].

[28] G. Aad et al. [ATLAS Collaboration], Phys. Lett. B 716, 1 (2012) arXiv:1207.7214 [hep-ex]].

[29] S. Chatrchyan et al. [CMS Collaboration], Phys. Lett. B 716, 30 (2012) arXiv:1207.7235 [hep-ex]].

[30] G. Aad et al. [ATLAS Collaboration], Phys. Rev. D 90 (2014) 11, 112015 arXiv:1408.7084 [hep-ex]].

[31] CMS Collaboration, Report No. CMS-PAS-HIG-14-009, July 2014.

[32] A. Djouadi, Phys. Rept. 457, 1 (2008) hep-ph/0503172. 